\title{
NOTE
}

\section{Effects of Diltiazem Hydrochloride on Pressor and Steroidogenic Actions of Angiotensins and Norepinephrine in Man}

\author{
Fumitake IKEDA $^{1}$, Tsuyoshi KONO ${ }^{1}$, Fumimaro OSEKO ${ }^{1}$, \\ HIROO IMURA ${ }^{1}$ AND JIRO ENDO ${ }^{2}$ \\ Second Division, Department of Internal Medicine, Kyoto University \\ School of Medicine, Shogoin, Sakyo-ku, Kyoto 606, Japan ${ }^{1}$, and \\ First Division, Department of Internal Medicine, Shimane \\ University School of Medicine, Izumo, Shimane \\ Prefecture 693, Japan ${ }^{2}$
}

\begin{abstract}
The effect of diltiazem hydrochloride (DTZ), a calcium-antagonist, on pressor and steroidogenic action of angiotensin II (AII), angiotensin III (AIII) and norepinephrine (NE) was studied in 5 normal men. AII, AIII and NE were infused intravenously for $50 \mathrm{~min}$ from $0900 \mathrm{hr}$ at a rate of $20 \mathrm{ng} / \mathrm{kg} / \mathrm{min}, 100 \mathrm{ng} / \mathrm{kg} / \mathrm{min}$ and $200 \mathrm{ng} / \mathrm{kg} / \mathrm{min}$, respectively. DTZ was infused intravenously alone or in combination with each of these pressor substances at a rate of $1 \mathrm{mg} / \mathrm{min}$ from $0910 \mathrm{hr}$ to $0930 \mathrm{hr}$. DTZ alone did not cause significant changes in blood pressure (BP) and plasma aldosterone concentration (Pl Aldo). On the other hand, DTZ combined with AII, AIII or NE significantly inhibited the rise in BP induced by these pressor substances. DTZ also inhibited the NE-induced increase in Pl Aldo, whereas it did not alter AII-, or AIIIinduced increase in $\mathrm{Pl}$ Aldo. These results indicate that in normal men pressor actions of AII, AIII and NE are calcium-dependent and calcium ions are also involved in NE-induced increase in P1 Aldo.
\end{abstract}

Angiotensin II (AII) and norepinephrine $(\mathrm{NE})$ are most potent pressor substances in animals and in men. However, the precise mechanism of their pressor actions is still unknown. It is known that Caions are the immediate trigger for the contraction of vascular smooth muscle. Recently inhibitory effects of diltiazem hydrochloride (DTZ), a Ca-antagonist, on pressor responses to AII and NE were reported in animals (Yamaguchi et al., 1974; Sato et al., 1978; Nagao et al., 1978). However, no data are available regarding the $\mathrm{Ca}$-dependecy of pressor and steroidogenic actions of AII, angiotensin III (AIII) and NE in man. The present paper is concerned with this point.

Received May 17, 1980.

\section{Experimental Subjects}

Five normal men examined in this study were 4 of the authors and a friend of theirs who had a good understanding of this experiment. Their ages were 32 to 54 years.

\section{Materials and Methods}

DTZ-d-cis-isomer of 3-acetoxy-2, 3-dihydro-5-[2(dimethylamino)ethyl]-2-(p-methoxy-phenyl)-1, 5-benzothiazepin- $4(5 \mathrm{H})$-one hydrochloride was provided by Tanabe Pharmaceutical Company, Osaka. AII([Asn ${ }^{1}$, $\mathrm{Val}^{5}$ ]ANG II) was provided by Ciba, Limited Osaka. AIII was confirmed by thin-layer chromatography. DTZ, AII and AIII were dissolved in, and NE was diluted with, physiological saline before use. The 5 
normal men examined were kept recumbent in the fasting condition after $0800 \mathrm{hr}$ on the day of the study. Sodium intake was not restricted until the night before the day of examination. However, it was estimated to be 170 to $200 \mathrm{mEq} / \mathrm{day}$ from sodium excretion data in 24 hour urine samples on the day before the examination. In our experience pressor and steroidogenic responses to AII and AIII do not vary significantly within this range of sodium intake.

Four experiments were done at one week intervals.

Experiment 1: DTZ was infused intravenously alone into the 5 normal men at the rate of $1 \mathrm{mg} / \mathrm{mun}$ from $0910 \mathrm{hr}$ to $0930 \mathrm{hr}$.

Experiment 2: AII was infused intravenously into the same 5 normal men at the rate of $20 \mathrm{ng} / \mathrm{kg} / \mathrm{min}$ from $0900 \mathrm{hr}$ to $0950 \mathrm{hr}$, and $1 \mathrm{mg} / \mathrm{min}$ of DTZ infusion was superimposed from $0910 \mathrm{hr}$ to $0930 \mathrm{hr}$.

Experiment 3: AIII was infused intravenously into the same 5 normal men at the rate of $100 \mathrm{ng}$ / $\mathrm{kg} / \mathrm{min}$ from $0900 \mathrm{hr}$ to $0950 \mathrm{hr}$, and $1 \mathrm{mg} / \mathrm{min}$ of DTZ infusion was superimposed from $0910 \mathrm{hr}$ to $0930 \mathrm{hr}$.

Experiment 4: NE was infused intravenously into the same 5 normal men at the rate of $200 \mathrm{ng} / \mathrm{kg} / \mathrm{min}$ from $0900 \mathrm{hr}$ to $0950 \mathrm{hr}$, and $1 \mathrm{mg} / \mathrm{min}$ of DTZ infusion was superimposed from $0910 \mathrm{hr}$ to $0930 \mathrm{hr}$.

The infusion volume of DTZ, AII, AIII, NE, DTZ plus AII, DTZ plus AIII and DTZ plus NE was always $0.3 \mathrm{ml} / \mathrm{min}$. Before, during and after the infusions of DTZ and these pressor substances, blood pressure (BP), heart rate (HR), plasma renin activity (PRA) and plasma aldosterone concentration (P1 Aldo) were measured. In Experiment 4, the plasma cortisol concentration (PC) was also determined. The radioimmunoassay methods for PRA, P1 Aldo and PC were described previously (Kono et al., 1975; Kono et al., 1980).

\section{Results}

Experiment 1: The results are shown in Fig. 1. Infusion of DTZ alone did not cause significant changes in BP, PRA and P1 Aldo.

Experiment 2: The results are shown in Fig. 2. Ten min after the beginning of AII infusion, BP rose remarkably and P1 Aldo showed a significant increase. HR and PRA showed significant decreases. Following the addition of DTZ infusion, BP began to fall within $2 \mathrm{~min}$ and showed a significant fall at $0930 \mathrm{hr}$, although not to the pretreatment level. HR increased to

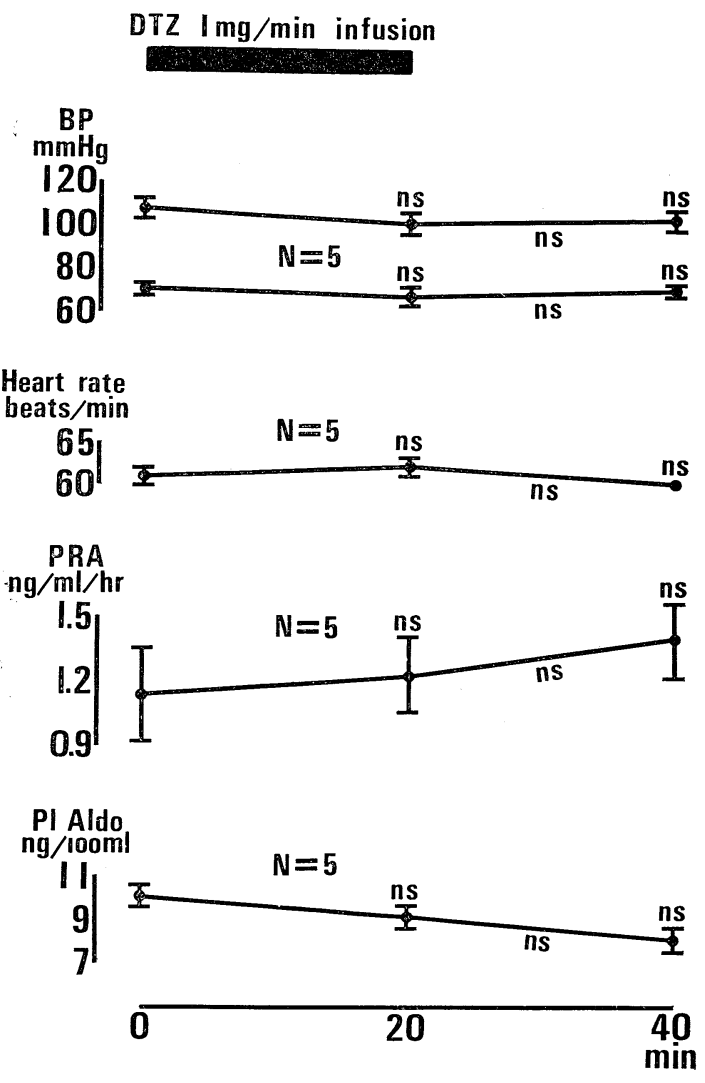

Fig. 1. Effect of $1 \mathrm{mg} / \mathrm{min}$ of diltiazem hydrochloride (DTZ) infusion on blood pressure (BP), heart rate, plasma renin activity (PRA) and plasma aldosterone concentration (Pl Aldo) in 5 normal men. Values are expressed as mean \pm SEM and statistically significant changes compared with pretreatment values or preceding values are shown by closed squares. "ns" expresses changes not statistically significant.

the pretreatment level, PRA showed no further change, and P1 Aldo showed a further significant increase. After the cessation of DTZ infusion, BP began to rise again but did not reach the level of 0910 hr. HR and Pl Aldo showed no further change, and PRA showed a further decrease.

Experiment 3: The results are shown in Fig. 3. Ten min after the beginning of AIII infusion, BP rose significantly and P1 


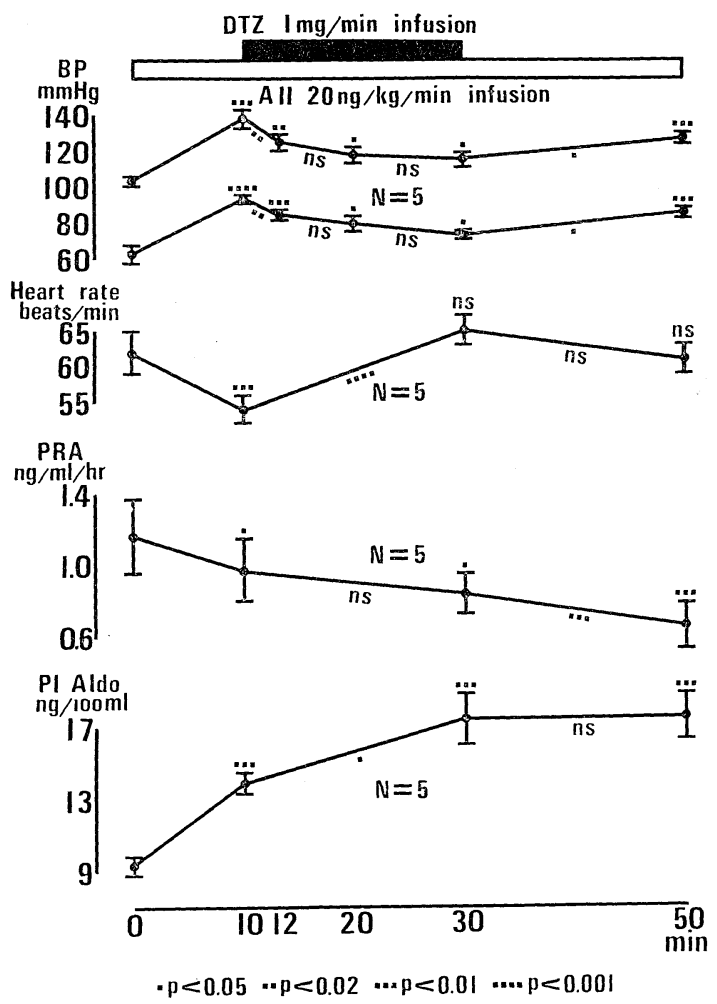

Fig. 2. Effect of $1 \mathrm{mg} / \mathrm{min}$ of diltiazem hydrochloride (DTZ) infusion on changes in blood pressure (BP), heart rate, plasma renin activity (PRA) and plasma aldosterone concentration (P1 Aldo) caused by $20 \mathrm{ng} / \mathrm{kg} / \mathrm{min}$ of angiotensin II (AII) infusion in 5 normal men. See legend for Fig. 1.

Aldo showed a significant increase. HR and PRA showed significant decreases. Following the addition of DTZ infusion, BP began to fall within $2 \mathrm{~min}$ and showed a significant fall at $0930 \mathrm{hr}$, although not to the pretreatment level. HR increased to the pretreatment level, PRA showed no further change, and P1 Aldo showed a further significant increase. After the cessation of the DTZ infusion, BP began to rise again but did not reach the level of $0910 \mathrm{hr}$. HR and PRA showed further significant decreases, and PlAldo showed a further significant increase.

Experiment 4: The results are shown

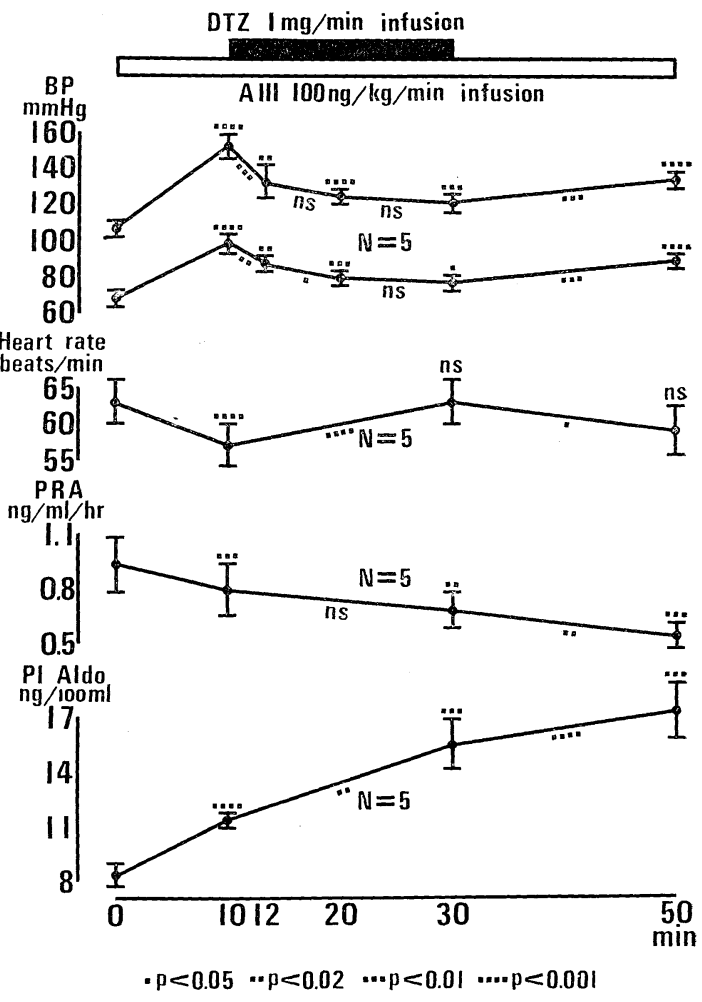

Fig. 3. Effect of $1 \mathrm{mg} / \mathrm{min}$ of diltiazem hydrochloride (DTZ) infusion on changes in blood pressure (BP), heart rate, plasma renin activity (PRA) and plasma aldosterone concentration (Pl Aldo) caused by $100 \mathrm{ng} / \mathrm{kg} / \mathrm{min}$ of angiotensin III (AIII) infusion in 5 normal men. See legend for Fig. 1.

in Fig. 4. Ten min after the beginning of NE infusion, BP rose significantly. HR showed a significant decrease, PRA and P1 Aldo showed significant increases, and PC showed no significant change. Following the addition of DTZ infusion, BP began to fall within $2 \mathrm{~min}$ and fell to the pretreatment level at $0930 \mathrm{hr}$. HR increased to the pretreatment level, PRA showed no further change, and Pl Aldo showed a significant decrease and reached a value lower than the pretreatment level. PC showed no significant change. After the cessation of the DTZ infusion, BP began to rise again but did not reach the level 


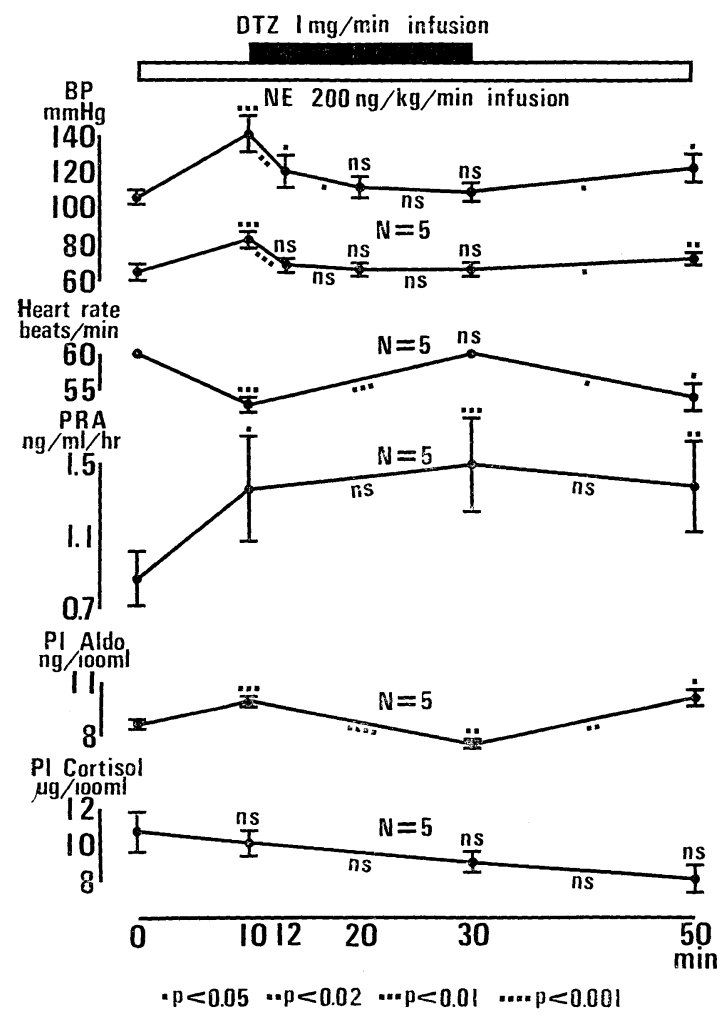

Fig. 4. Effect of $1 \mathrm{mg} / \mathrm{min}$ of diltiazem hydrochloride (DTZ) infusion on changes in blood pressure (BP), heart rate, plasma renin activity (PRA), plasma aldosterone concentration ( $\mathrm{Pl}$ Aldo) and plasma cortisol concentration (Pl Cortisol) caused by $200 \mathrm{ng} / \mathrm{kg} / \mathrm{min}$ of norepinephrine (NE) infusion in 5 normal men. See legend for Fig. 1.

of $0910 \mathrm{hr}$. HR showed a significant decrease, and PRA and PC showed no significant changes. Pl Aldo showed a significant increase and the value at $0950 \mathrm{hr}$ was similar to that at $0910 \mathrm{hr}$. In all of the 4 experiments, serum sodium and serum potassium did not change significantly.

\section{Discussion}

Contraction of muscle cells requires an increase in the intracellular concentration of free $\mathrm{Ca}$ ions, and $\mathrm{Ca}$-antagonists are thought to be effective as vasodilators by inhibiting the influx of $\mathrm{Ca}$ ions through the cell membranes of cardiac and vascular smooth muscles and/or by inhibiting the release of free $\mathrm{Ca}$ ions from $\mathrm{Ca}$ storage in these smooth muscle cells (Grün and Fleckenstein, 1972; Fleckenstein et al., 1972). DTZ, one of the Ca-antagonists which was synthesized in Japan (Sato et al., 1971), has been used as a coronary vasodilator for the therapy of coronary arterial spasm (Yasue et al., 1978). It also has some hypotensive action in dogs (Ishikawa et al., 1978). DTZ inhibits NE-induced contraction of the aortic strip of the rabbit (Nagao et al., 1978) and pressor actions of AII and $\mathrm{NE}$ in rats (Sato et al., 1978). In dogs it inhibits renal vasoconstricting action of AII whereas it has no effect on that of epinephrine (Yamaguchi et al., 1974). However, these results were obtained in animals, and there has been no report on the effects of Ca-antagonists on the actions of AII, AIII and NE in man. This encouraged us to undertake the present experiments.

The doses of AII, AIII and NE administered in the present study were known to be tolerable and sufficient enough to cause a significant rise in BP (Kono et al., 1975; Kono et al., 1976; Kono et al., 1978). In the present experiments AII-, AIII- and NE-induced BP rises were all inhibited by the combined infusion of DTZ, whereas DTZ alone snowed no hypotensive effect in 5 normal men. This suggests that the pressor actions of AII, AIII and NE in normal men are Ca-dependent. This inhibition was incomplete when DTZ was combined with AII or AIII and was complete when DTZ was combined with NE, and some inhibitory effects on pressor actions of these pressor substances still remained even $20 \mathrm{~min}$ after the cessation of the DTZ infusion.

HR decreased when BP rose, whareas it increased when BP fell in the Experiments 2,3 and 4 . This may be due to a cardiovascular reflex. 
PRA decreased after AII or AIII infusion alone. This may be due to negative feedback inhibition resulting from BP rise or/and direct inhibition of renin release by these angiotensins (Kono et al., 1976; Steele et al., 1976; Kono et al., 1978). On the other hand, PRA increased after NE administration alone in spite of a significant BP rise. This increase in PRA may be due to stimulation of adrenergic receptors in the juxtaglomerular apparatus. Generally beta receptors are thought to mediate the increase in renin release (Assaykeen et al., 1970). However, it is also shown that both $\mathrm{NE}$, an alpha-adrenergic stimulator, and isoproterenol, a beta-adrenergic stimulator, increase renin secretion (Winer et al., 1971). When DTZ was added in Experiments 2, 3 and 4, PRA showed no further changes. The reason for this is unknown, because DTZ alone had no effect on PRA. There is a diurnal variation in PRA during constant recumbency (Katz et al., 1972). However, it is unlikely that significant changes in PRA occur between $0900 \mathrm{hr}$ and $0950 \mathrm{hr}$.

P1 Aldo increased after AII, AIII and $\mathrm{NE}$ infusion alone. Increases in Pl Aldo after AII and AIII are thought to be caused exclusively by direct stimulation of adrenal zona gromerulosa cells by the angiotensins and not by ACTH stimulation, because in our previous studies PC did not increase after infusion of the same doses of AII and AIII in normal men (Kono et al., 1975; Kono et al., 1978). In Experiments 2 and 3 , the addition of DTZ did not inhibit further increase in $\mathrm{Pl}$ Aldo. It was reported recently that in an in vitro experiment using isolated glomerulosa cells of rats the aldosterone production induced by $\mathrm{ACTH}, \mathrm{AII}$ and potassium was Ca-dependent and reduced by decreasing the extracellular $\mathrm{Ca}$ concentrations (Fakunding et al., 1979). But, unlike muscle cells, it is not known whether or not Ca-antagonists influence the influx or outflux of $\mathrm{Ca}$ ions through the cell membranes of adrenal glomerulosa cells in vivo. If they have this effect the present results seem to suggest that $\mathrm{Ca}$ ions are not involved in tne AIIond AIII-induced increases in PI Aldo in normal men. However, DTZ infusion was superimposed for only $20 \mathrm{~min}$ in the present experiments. This duration was too short to assess an inhibitory effect of DTZ on aldosterone stimulation when P1 Aldo did not decrease, because the biological half-life of aldosterone in the blood plasma of normal subjects is about $25 \mathrm{~min}$ (Kono et al., 1966) and at $0930 \mathrm{hr}$ prestimulated. level of Pl Aldo may still persist even if DTZ exerts some inhibitory influence upon subsequent aldosterone stimulation by AII and AIII. Therefore, we cannot conclude from the present results that AII- and AIIIinduced aldosterone stimulation are not $\mathrm{Ca}$ dependent. In a previous report an increase in aldosterone secretion was not consistently observed in normal subjects after 8- to 24hour infusions of NE (Ames et al., 1965). However, in the present study an increase in P1 Aldo $10 \mathrm{~min}$ after the beginning of the NE infusion was slight but statistically significant. The cause of this increase in Pl Aldo is unknown. It is clear that this increase in Pl Aldo is not due to ACTH stimulation, because PC showed no increase at this point. Of great interest was a significant decrease in P1 Aldo when DTZ was added to NE. Moreover, when DTZ was removed, Pl Aldo began to increase again. On the other hand, PRA showed no significant change when DTZ was added to NE and when it was removed later. This non-parallelism of PRA and Pl Aldo indicates that the NE-induced increase in Pl Aldo is not secondary to an increase in PRA. PC also showed no significant change during the addition of DTZ to NE and the removal of DTZ. These results suggest that $\mathrm{Ca}$ ions are involved in the NE-induced increase in Pl Aldo, although the precise mechanism is unknown. $\mathrm{Ca}$ 
ions are thought to be a second messenger in the actions of several hormones including ACTH. However, from the present results, it is evident that the decrease in P1 Aldo by the addition of DTZ to NE is not caused by ACTH suppression. There is a diurnal variation in $\mathrm{Pl}$ Aldo during constant recumbency (Katz et al., 1972). However, it is unlikely that P1 Aldo changes significantly between $0900 \mathrm{hr}$ and $0950 \mathrm{hr}$.

\section{Acknowledgments}

The authors are grateful to the staff of Tanabe Pharmaceutical Company, Osaka for kindly supplying DTZ for the present study.

\section{References}

Ames, R. P., A. J. Borkowski, A. M. Sicinski and J. H. Laragh (1965). J. Clin. Invest. 44, 1171.

Assaykeen, T. A., P. L. Clayton, A. Goldfien and W. F. Ganong (1970). Endocrinology 87, 1318.

Fakunding, J. L., R. Chow and K. J. Catt (1979). Ibid. 105, 327.

Fleckenstein, A., H. Tritthart, H. J. Döring and K. Y. Byon (1972). Arzneim. Forsch. 22, 22.

Grün, G. and A. Fleckenstein (1972). Ibid. 22, 334. Ishikawa, H., M. Matsushima, H. Matsui, A. Honjo,
M. Hayashi, T. Shindo, T. Morifuji and M. Okabayashi (1978). Ibid. 28, 402.

Katz, F. H., P. Romfh and J. A. Smith (1972). J. Clin. Endocrinol. 35, 178.

Kono, T., F. Ikeda, F. Oseko, H. Imura and J. Endo (1980). Ibid. 50, 40.

Kono, T., F. Ikeda, F. Oseko, M. Nanno, H. Imura and J. Endo (1978). Acta Endocrinol. (Kbh.) 87, 359.

Kono, T., F. Oseko, S. Shimbo, M. Nanno and J. Endo (1975). J. Clin. Endocrinol. 41, 1174.

Kono, T., F. Oseko, S. Shimbo, M. Nanno, F. Ikeda and J. Endo (1976). Ibid. 43, 692.

Kono, T., T. Yoshimi and T. Miyake Steroid Dynamics (edited by G. Pincus, T. Nakao and J. F. Tait). Academic Press, New York and London, p. 429 (1966).

Nagao, T., T. Ikeo, M. Sato, H. Nakajima and A. Kiyomoto Recent Advances in Studies on Cardiac Structure and Metabolism, Vol. 11 (edited by T. Kobayashi, T. Sano and N. S. Dhalla). University Park Press, Baltimore, p. 437 (1978).

Sato, M., T. Nagao, I. Yamaguchi, H. Nakajima and A. Kiyomoto (1971). Arzneim. Forsch. 21, 1338.

Sato, R., S. Sawada and S. Tanaka (1978). Jpn. Circ. J. 42, 571.

Steele, J. M., A. J. Neusy and J. Lowenstein (1976). Circ. Res. 38 (Suppl. II), 113.

Winer, N., D. S. Choski and W. G. Walkenhorst (1971). Circ. Res. 29, 239.

Yamaguchi, I., K. Ikezawa, T. Takeda and A. Kiyomoto (1974). Jpn. J. Pharmacol. 34, 511.

Yasue, H., M. Nagao, S. Omote, A. Takizawa, K. Miwa and S. Tanaka (1978). Circulation 58, 56. 